\title{
SMALL-SIZED MAGNESIUM CYLINDERS INFLUENCE SUBCHONDRAL BONE QUALITY IN OSTEOARTHRITIC RABBITS - AN IN VIVO PILOT STUDY
}

\author{
N. Angrisani ${ }^{1, *}$, R. Willumeit-Römer ${ }^{2}$, H. Windhagen ${ }^{1}$, B. Mavila Chathoth ${ }^{2}$, V. Scheper ${ }^{3}$, B. Wiese ${ }^{2}$, \\ H. Helmholz ${ }^{2}$ and J. Reifenrath ${ }^{1}$
}

\begin{abstract}
${ }^{1}$ Hannover Medical School, Clinic for Orthopaedic Surgery, NIFE-Lower Saxony Centre for Biomedical Engineering, Implant Research and Development, Hannover, Germany

${ }^{2}$ Helmholtz-Zentrum Hereon, Institute of Metallic Biomaterials, Geesthacht, Germany

${ }^{3}$ Hannover Medical School, Department of Otolaryngology, NIFE-Lower Saxony Centre for Biomedical Engineering, Implant Research and Development, Hannover, Germany
\end{abstract}

\begin{abstract}
No optimal therapy exists to stop or cure chondral degeneration in osteoarthritis (OA). While the pathogenesis is unclear, there is consensus on the etiological involvement of both articular cartilage and subchondral bone. Compared to original bone, the substance of sclerotic bone is mechanically less solid. The osteoproliferative effect of $\mathrm{Mg}$ has been shown repeatedly during development of $\mathrm{Mg}$-based osteosynthesis implants. The aim of the present study was to examine the influence of implanted high-purity Mg cylinders on subchondral bone quality in a rabbit OA model. $10 \mathrm{New}$ Zealand White rabbits received into the knee either 20 empty drill holes or 20 drill holes, which were additionally filled with one Mg cylinder each. Follow-up was at 8 weeks. Micro-computed tomography $(\mu \mathrm{CT})$ was performed. After euthanasia, cartilage condition was determined, bone samples were collected and processed for histological evaluation and elemental imaging by micro-Xray fluorescence spectrometry $(\mu \mathrm{XRF})$.

Articular cartilage collected post-mortem showed different stages of lesions, from mild alterations up to exposed subchondral bone, which tended to be slightly lower in animals with implanted Mg cylinders. $\mu \mathrm{CT}$ showed significantly increased bone volume in the Mg group. Also, histological evaluation revealed distinct differences. While right, operated limbs did not show any significant difference, left, non-operated controls showed significantly less changes in articular cartilage in the Mg group.

A distinct influence of implanted cylinders of pure $\mathrm{Mg}$ on subchondral bone of osteoarthritic rabbits was shown. Subsequent evaluations, including other time points and alternative alloys, will show if this could alter OA progression.
\end{abstract}

Keywords: In vivo, cartilage, bone structure, bone density, osteoarthritis progression, magnesium, degradation.

*Address for correspondence: Dr Nina Angrisani, Medizinische Hochschule Hannover, Stadtfelddamm 34, 30625 Hannover, Germany.

Telephone number: +495115328961 Email: angrisani.nina@mh-hannover.de

Copyright policy: This article is distributed in accordance with Creative Commons Attribution Licence (http://creativecommons.org/licenses/by-sa/4.0/).

\begin{tabular}{llll}
\hline & List of Abbreviations & IPL & image processing language \\
& & OA & osteoarthritis \\
AAS & atomic absorption spectrometry & ROI & region of interest \\
ACLT & anterior crucial ligament transection & SBP & subchondral bone plate \\
ANOVA & analysis of variance & s.c. & subcutaneously \\
AP & alkaline phosphatase & SEM & scanning electron microscopy \\
BD & bone density & tr.th. & trabecular thickness \\
BV & bone volume & $\alpha-\mathrm{MEM}$ & alpha-minimum essential medium \\
bw & body weight & $\mu C T$ & micro-computed tomography \\
FBS & foetal bovine serum & $\mu$ XRF & micro-X-ray fluorescence \\
HA & hydroxyapatite & & spectrometry
\end{tabular}




\section{Introduction}

OA is one of the prevailing diseases in the orthopaedic field. With a prevalence of $80 \%$ in patients older than 75 years (Arden and Nevitt, 2006), this degenerative joint disease has further gained in importance due to an increasing life expectancy while obesity is also more common (Web ref. 1). Although the exact pathogenesis of OA is still not fully clarified, there is consensus about the aetiological involvement of both articular cartilage and subchondral bone and their interconnection via signalling cascades. The lifetime of a prosthesis as well as the feasible number of revision surgeries are limited. Therefore, all possibilities to preserve the joint should be exhausted before partial or total replacement and focus should be on therapies to slow down degenerative processes in earlier stages of OA.

At present, no optimal therapy exists to stop or cure cartilage degeneration. Depending on size of lesion and degree of pain, different therapeutical approaches are exerted. In smaller defects, damaged cartilage is removed and regenerative cells transferred to the defect site by e.g. debridement, spudding or micro-fracturing of the SBP to initiate migration of stem cells and growth factors and therewith influence positively cartilage and bone healing (Oliver-Welsh et al., 2016).

An essential aspect of progressive degeneration is the change in the subchondral bone. First, increased remodelling of bone occurs and therewith loss of subchondral bone matrix. Subsequently, presumably stress-induced sclerotisation of SBP starts. Although a sclerotic bone shows higher $\mathrm{BV}$, mineralisation is decreased, making the sclerotic bone a mechanically less stable tissue (Grynpas et al., 1991). Experimental studies showed that chondral quality in OA models correlate with quality/stability of subchondral bone (Acar et al., 2016; Bellido et al., 2011). Therefore, a possible therapeutical approach is to influence the stability of the SBP and formation of cartilaginous replacement tissue.

$\mathrm{Mg}$ is one of the mass elements of the body and essential for cells and enzymes. In recent years, Mg-based implants have gained much interest in orthopaedics as they degrade during e.g. fracture healing, thus making redundant second surgeries to remove implants. Besides good biocompatibility and the need to carefully control degradation rates due to associated gas accumulation, numerous studies have demonstrated the osteoproliferative effect of $\mathrm{Mg}$ or its degradation products, as summarised in a recent review by Willbold et al. (2017). One of the first reports on increased mineral apposition rate and $\mathrm{BV}$ around $\mathrm{Mg}$ rods in vivo was published by Witte et al. (2005). They examined four different $\mathrm{Mg}$ alloys in guinea pigs, with the conclusion of a possible bone cell activation by $\mathrm{Mg}$ ions. Kraus et al. (2012) demonstrated a degradation-rate-dependent increase in BV in a rat model and Janning et al. (2010) showed a temporarily stimulating effect of $\mathrm{Mg}$ hydroxide cylinders on osteoblast activity in rabbits while osteoclast activity was reduced. A comparable effect was observed with an Ag-containing material (Mg$2 \mathrm{Ag}$ ) improving osteoblast activity in vitro as well as in vivo in a mice fracture model (Jahn et al., 2016). These reports, comprising a broad range of animal models, demonstrate the high certainty of the stated osteoproliferative effect of $\mathrm{Mg}$. However, not only $\mathrm{BV}$ can be influenced by Mg-based materials but also bone quality. The effect of Mg-based materials on differences in mineralisation, orientation and thickness of the HA are assessed utilising smallangle X-ray scattering and X-ray diffraction (ZellerPlumhoff et al., 2020). Nygren et al. (2017) showed an increased production of HA during bone remodelling near MgO implants (Nygren et al., 2017). They presumed a $\mathrm{pH}$-triggered activation of nociceptors accompanied by the release of neuropeptide substance $\mathrm{P}$, resulting in the increased production of HA. Grünewald et al. (2016) showed that $\mathrm{Mg}$ accumulation near the implant interface led to an altered HA structure through substitution of $\mathrm{Ca}$ ions by $\mathrm{Mg}$ ions. The altered structure was characterised by smaller mineralised bone plates. Areas with elevated Mg levels showed increased mechanical stability when compared to areas with lower $\mathrm{Mg}$ levels, which was presumed to be the result of the smaller crystalline structures (Grünewald et al., 2016).

Previous studies have used Mg implants as supportive structures, which need a sufficient stability and provide proper fixation of bone parts while additionally enhancing/accelerating fracture healing. The aim of the present study was to use the osteoproliferative capacity of $\mathrm{Mg}$ and its positive effect to reinforce bone structural parameters and influence the biomechanical properties of the SBP, therewith attenuating the progress of cartilage degeneration and promoting fibrocartilage regeneration. In this context, small-sized cylinders would allow for a precise positioning using press-fit technique and would avoid the necessity for large scale surgical defects within cartilage and bone, thus preserving as much original tissue as possible. The study hypothesis was that uniformly distributed, small subchondrally implanted Mg cylinders induced increased mineralisation of the SBP and underlying trabeculae and enhanced conditions favourable for cartilage in osteoarthritic rabbits when compared to animals without implanted $\mathrm{Mg}$. The pilot study should provide proof of the hypothesis as well as basic results for proper a priori sample size calculations of subsequent studies.

\section{Material and Methods}

\section{Mg wire}

Pure Mg (99.985 \%, MAGONTEC, Sydney, Australia) was casted using the permanent direct chill casting process. The ingot was machined into cylinders with a diameter of $49 \mathrm{~mm}$ and a length of $150 \mathrm{~mm}$ for the 
Table 1. Element composition of pure bulk $\mathrm{Mg}$ in $\mathrm{mg} / \mathrm{kg}$.

\begin{tabular}{|c|c|c|c|}
\hline Impurity & $\mathrm{Fe}(\mathrm{mg} / \mathrm{kg})$ & $\mathrm{Cu}(\mathrm{mg} / \mathrm{kg})$ & $\mathrm{Ni}(\mathrm{mg} / \mathrm{kg})$ \\
\hline Content & $20 \pm 2$ & $15 \pm 1$ & $13 \pm 3$ \\
\hline
\end{tabular}

direct extrusion process. Prior to the extrusion at $350{ }^{\circ} \mathrm{C}$, the billet was preheated for $1 \mathrm{~h}$. The extrusion was carried out at an extrusion speed of $0.11 \mathrm{~mm} / \mathrm{s}$ and using a die with four $0.8 \mathrm{~mm}$ diameter holes. The cold wire drawing was conducted with a step size of $0.05 \mathrm{~mm}$ and a recrystallisation annealing of $5 \mathrm{~min}$ at $350{ }^{\circ} \mathrm{C}$ per step until the final diameter of $0.5 \mathrm{~mm}$ was obtained. Impurities of the material, measured by spark spectrometry, are shown in Table 1.

\section{Mg cylinder processing and cleaning}

Cylindrical implants were made from $\mathrm{Mg}$ wires by punching using an age-hardened Mg-4Y-3Gd punching tool. Each punch yielded 60 small cylinders of the same size and geometry. The average length and diameter of the cylinders $(n=26)$ was $957.5 \pm 5.5$ and $519.5 \pm 2.5 \mu \mathrm{m}$, respectively, as determined by optical microscopy (Leica Wild M3Z). The cylinders were then subjected to the cleaning procedure described by Nidadavolu et al. (2016), utilising an ultrasonic bath for 20 min - using n-hexane followed by acetone. A further cleaning step was performed using $100 \%$ ethanol to remove any traces of impurities, if present. A final sterilisation step was performed using $70 \%$ ethanol.

\section{Microstructure and shape}

For the microstructure investigations, the small cylinders were embedded in epoxy resin (Demotec $30^{\circledR}$, Demotec Demel e.K, Nidderau, Germany) and ground using 2,500 grit $\mathrm{SiC}$ paper at a speed of $80 \mathrm{rpm}$. The investigated directions were longitudinal and transverse to the drawing direction. For both directions, samples were ground to the centre of the implants. Finally, samples were polished using water containing oxide polishing suspension, a $\mathrm{SiO}_{2}$ solution (O.P.S. Oxid-Polier-Suspension, SchmitzMetallographie GmbH, Herzogenrath, Germany), at a speed of $80 \mathrm{rpm}$. Samples were etched using a picric acid solution (Kree et al., 2004) and observation of the microstructure was performed using an optical microscope (Leica Wild M3Z).

The shapes were investigated by SEM (TESCAN VEGA3-SB, Brno, Czech Republic) using the secondary electron detector and an accelerating voltage of $20 \mathrm{kV}$. For this purpose, the cylinders were attached to a specimen holder with an electrically conductive sticker.

\section{Degradation-profiling immersion test}

Degradation rates of $\mathrm{Mg}$ cylinders were determined by a weight-loss method. Cylinders were accurately weighed to $10 \mathrm{mg}$ and inserted into transwells of 24 well plates (Greiner Bio-One, ThinCerts, pore size $3 \mu \mathrm{m})$. Samples were treated with $500 \mu \mathrm{L}$ of $\alpha$-MEM (Life Technologies) supplemented with
$10 \%$ FBS (PAA Laboratories). Pure, complete cell culture medium served as control. Experiments were performed for $3,7,14,21$ and $28 \mathrm{~d}$ under conditions of normoxia ( $20 \%$ oxygen) and hypoxia (3\% oxygen), respectively (Ahmad et al., 2016). Long-term tests were carried out for 6 and 8 weeks adopting similar procedure. Medium was replaced every 2-3 d for $\mathrm{pH}$ maintenance. Replaced media were measured for changes in $\mathrm{pH}$ (SENTRON ARGUS X pH-meter, Fisher Scientific) and osmolality (Osmomat 030, Gonotec, Berlin, Germany). After the endpoint of experiment (3, 7, 14, 21 and $28 \mathrm{~d} ; 6$ and 8 weeks), samples were washed 3 times with subsequent washes of water and ethanol (100\%). Later, the degradation layers developed on the samples were removed using fresh chromic acid $(180 \mathrm{~g} / \mathrm{L}$ in distilled water) placed in direct contact with the samples for $20 \mathrm{~min}$. All experiments were performed in 6 replicates. Weights of sample sets, before and after chromic acid treatments, were determined using a highly accurate weighing scale (RADWAG Wagi Electroniczne, Hilden, Germany; model: MYA 2.4Y, $\mathrm{S} / \mathrm{N}: 537265$, precision $1.5 \mu \mathrm{g})$. The mean degradation depths (h) of the samples were calculated using the equation below (1).

$$
h=\frac{\Delta m \times f}{A \times \rho}
$$

where $h$ is the mean degradation depth in $\mu \mathrm{m}, \Delta m$ is the weight loss in $g, f$ is the unit conversion factor $(10,000)$ to obtain mean degradation depth in $\mu \mathrm{m}$, $A$ is the surface area of the sample in $\mathrm{cm}^{2}, \rho$ is the density in $\mathrm{g} / \mathrm{cm}^{3}$. The cylinders were imaged by optical microscopy (Leica WILD M3Z) to qualitatively evaluate the degradation of surface morphology. Quantification of $\mathrm{Mg}$ concentration released into the medium was measured by atomic absorption spectrometry (AAS-Agilent 240 AA) utilising the standard instrument method for $\mathrm{Mg}$. An external calibration was performed for quantification, which utilises an Mg reference solution (Roth single Element standard for AAS) to cover the targeted analytic range: $0.1-1.0 \mathrm{mg} / \mathrm{L}$ ). According to the standard range, the media containing $\mathrm{Mg}$ samples were diluted with $1 \% \mathrm{HNO}_{3}$ in ultrapure water type I. Measurements were recorded for 3 and $5 \mathrm{~d}$, respectively.

\section{Animal study}

The in vivo experiments were performed according to the German Animal Welfare act (registration number 33.9-42502-04-18/2774) using 10 adult, female New Zealand White rabbits with an average bw of $3.46 \pm 0.23 \mathrm{~kg}$ (Charles River Laboratories, Research Models and Services $\mathrm{GmbH}$ ). The rabbits were housed in groups of minimum 3 animals and allowed free movement. At the beginning of the 
study, they were $>30$ weeks old. In an initial surgery, all animals underwent ACLT in combination with an incision of the medial meniscus so as to induce OA. Anaesthesia was initiated by an intramuscular injection of ketamine $(20 \mathrm{mg} / \mathrm{kg}$ bw, ketamine $10 \%$, CP-Pharma, Burgdorf, Germany) and medetomidine (0.25 mg/kg bw, Cepetor, CP-Pharma). After reaching unconsciousness, the rabbits were intubated and a peripheral venous catheter was placed into the vena auricularis. The right hind limb was clipped, washed and disinfected and the animals were placed in dorsal recumbency. Rabbits received Bepanthene ${ }^{\circledR}$ eye ointment to avoid eye desiccation. Meloxicam $(0.15 \mathrm{mg} / \mathrm{kg}$ bw, s.c., Metacam injection solution, Boehringer Ingelheim) and fentanyl (0.005 mg/ kg bw, s.c., Janssen-Cilag, Neuss, Germany) were administered for peri-operative analgesia. Anaesthesia maintenance was performed by 2.5-4\% isoflurane inhalation in oxygen mixture. Skin and knee capsules were incised medially and the anterior cruciate ligament was exposed. Transsection of the ligament and incision of the medial meniscus were performed according to Pauly et al. (2015). Afterwards, the joint capsule and skin were closed layer by layer using Vicryl ${ }^{\circledR}$ 4/0 (Ethicon/Johnson\&Johnson). To reduce the postoperative recovery time, atipamezole (0.5-1.0 mg/kg bw, Revertor, CP-Pharma) was injected intramuscularly. Postsurgical medication comprised meloxicam administration (Metacam ${ }^{\circledR}$ oral suspension, $20 \mathrm{mg} / \mathrm{kg}$ bw, per os) as analgesia for $7 \mathrm{~d}$ and enrofloxacin injection (Baytril ${ }^{\circledR}, 10 \mathrm{mg} / \mathrm{kg} \mathrm{bw}$, s.c.) as infection prophylaxis for $5 \mathrm{~d}$. During 12 weeks following the first surgery, animals developed OA.

For the second surgery, they were assigned randomly by weight to one of two groups with $n=5$ rabbits each so that mean bw in both groups was similar. Group 1 got drilling holes only (group "drilling"), group 2 received $\mathrm{Mg}$ cylinders into the primarily placed drilling holes (group " $\mathrm{Mg}^{\prime \prime}$ ). Anaesthesia and analgesic procedure were similar to the first surgery. Opening of the joint was performed through the same approach as in the ACLT. The subpatellar fat pad was partially removed to enable sufficient access to medial and lateral condyles. In the lateral and medial compartment of both, femur and tibia, 5 holes each with a diameter of $0.5 \mathrm{~mm}$ were drilled using a sterilisable, battery-driven hand-held drill (AccuPen, RISystem AG), summing up to a total of 20 holes per animal. After drilling, animals of group $\mathrm{Mg}$ additionally received one cylindrical $\mathrm{Mg}$ cylinder per drill hole. Implants were inserted immediately following drilling using a custom-made target device that comprised of shortened $20 \mathrm{G}$ and $25 \mathrm{G}$ syringes. While $20 \mathrm{G}$ syringes served as guiding tubes for the cylindrical implants, $25 \mathrm{G}$ syringes were applied as stylets to insert the cylinders at a reproducible depth in the subchondral bone. Fig. 1 depicts important steps of the insertion (Fig. 1). After placement of
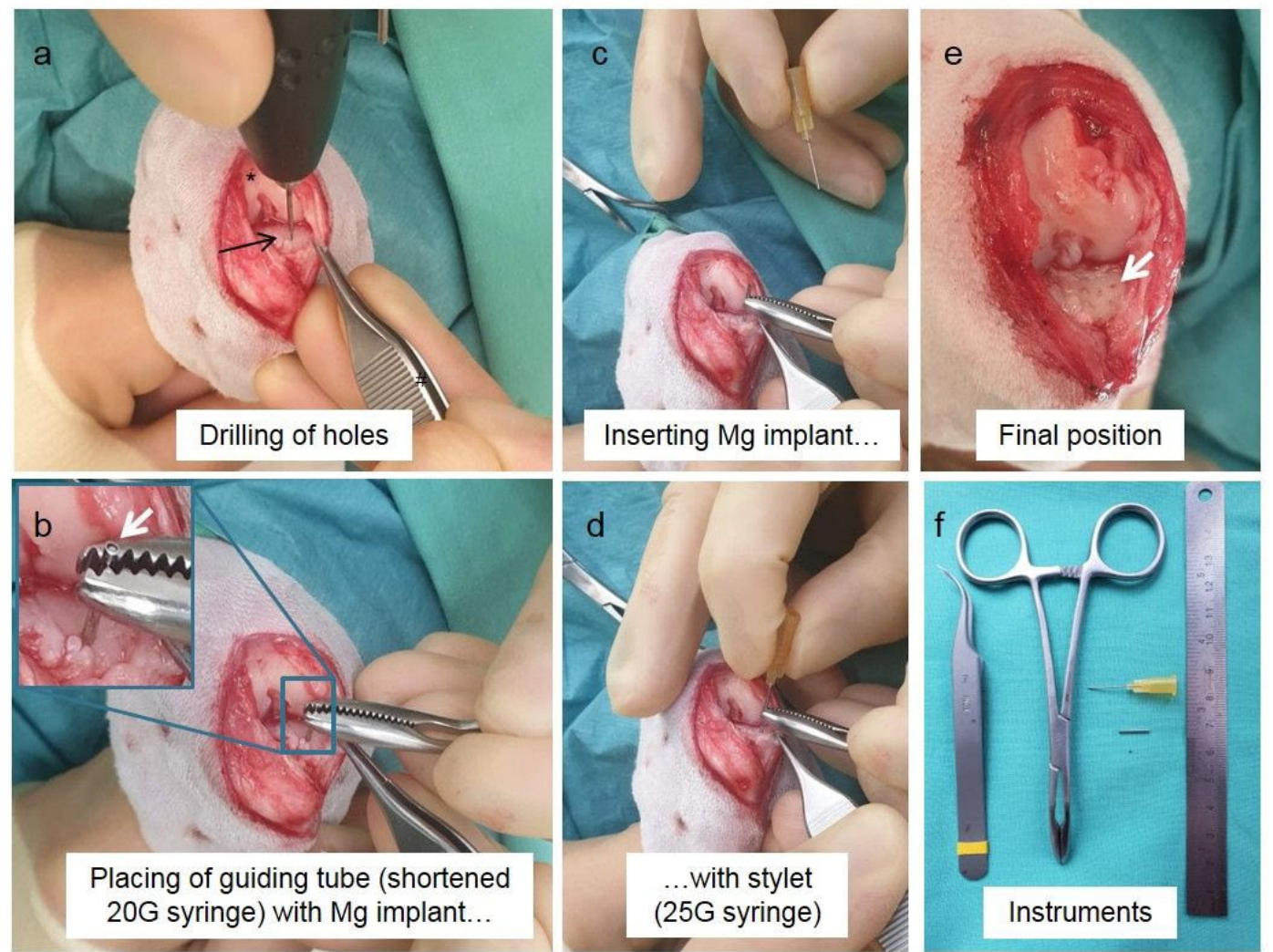

Fig. 1. Surgical procedure. (a) Placement of drilling holes (drilling group and Mg group). (b) After drilling of the holes, the guiding tube already containing the previously inserted $\mathrm{Mg}$ cylinder (white arrow in magnification image) was placed over the hole. (c,d) Insertion of $\mathrm{Mg}$ cylinder into the subchondral bone using a stylet. (e) Final position of holes with or without $\mathrm{Mg}$, here for example in the medial tibia compartment (white arrow). Asterisk: femur; black arrow: tibia. 
Table 2. Score values for the evaluation of cartilage changes and occurrence of osteophytes in the knee joint after euthanasia.

\begin{tabular}{|c|c|c|}
\hline \multirow{4}{*}{ Cartilage changes } & 0 & Surface smooth, with normal cartilage \\
\cline { 2 - 3 } & 1 & Surface rough, with minimal fibrillation or slight yellowish discoloration \\
\cline { 2 - 3 } & 2 & Cartilage erosion extending into the superficial or middle layers \\
\cline { 2 - 3 } & 3 & Cartilage erosion extending into the deep layers \\
\cline { 2 - 3 } & 4 & Complete cartilage erosion, with subchondral bone exposed \\
\hline \multirow{5}{*}{ Osteophytes } & 0 & Absent \\
\cline { 2 - 3 } & 1 & Mild \\
\cline { 2 - 3 } & 2 & Moderate \\
\cline { 2 - 3 } & 3 & Severe \\
\hline
\end{tabular}

either drill holes alone or drill holes and Mg implants, loose tissue originating from OA changes (menisci, cartilage, synovium) was removed carefully and the joint capsule was closed (Vicryl ${ }^{\circledR} 4 / 0$, Ethicon/ Johnson\&Johnson). Joint instability caused by the ACTL was fixed by extracapsular fixation according to van Zuijlen et al. (2010). In brief, one suture loop each was fixed around the medial and lateral fabella (Ethibond 2, Ethicon/Johnson\&Johnson). Then, sutures were directed distally across the joint without penetrating the capsule and crossed through a tunnel that was drilled slightly distal to the tuberositas tibiae. Suture knots were placed at both sides lateral of the patella. Postsurgical medication was similar to the first surgery.

\section{Exclusion criteria}

Animals who had critical complication not related to the study (e.g. anaesthetic accidents) were excluded from the study and replaced by new animals. If not stated otherwise, all animals were included in the single analyses.

All evaluations and scorings were performed with the assessor unaware of the group affiliation of the respective samples.

\section{Clinical assessment, blood sampling}

Animals were examined daily during the first 2 weeks following each surgery and, subsequently, 3 times a week. Evaluation was performed using a score system including the following parameters: weight reduction, general condition, spontaneous behaviour and wound healing (redness, swelling and adaption of suture). Special orthopaedic scoring comprised of pain detection during passive motion and touching and weight-bearing ability during active motion and resting. Score values ranged from 0 for no pathological changes to 10 (passive/active pain) resp.20 (weight, behaviour, wound healing) as most possible pathological changes.

At the day of the second surgery as well as during anaesthesia for both following $(\mu \mathrm{CT})$ scans, serum blood samples were taken from each rabbit. The tubes were centrifuged, serum transferred to cryotubes and stored at $-80^{\circ} \mathrm{C}$ until further evaluation. Blood samples were evaluated for AP as an enzymatic marker for bone metabolism by an accredited analysis laboratory (LABOKLIN, Bad Kissingen, Germany).

\section{$\mu \mathrm{CT}$}

For the detection of quantitative and qualitative changes in subchondral bone and trabeculae, both knees of each rabbit were scanned using a $\mu \mathrm{CT}$ scanner (XtremeCTII, ScancoMedical AG). The scans were performed immediately after the second surgery (application of drill holes $/ \mathrm{Mg}$, scan 1), as well as after 4 (scan 2) and 8 weeks (scan 3) using the anaesthetic protocol described in the "Animal study" paragraph. Animals were placed in a special carbonmade sample/animal tube in dorsal recumbency. Both feet were fixed at the end of the tube and knees were placed parallel to each other. Knees were scanned from the beginning of the patella to the region of the tuberositas tibiae using $353 \mathrm{mAs}, 42.7 \mathrm{mGy}$ at an integration time of $103 \mathrm{~ms}$, resulting in a resolution of $30 \mu \mathrm{m}$. After all scans were performed, data of second and third scans were registered and transformed to the first scan to align all bones and allow for evaluation of equal regions over time. Therefore, the specific region of the femur and tibia, respectively, were contoured manually and aligned using the software IPL (ScancoMedical AG). Afterwards, the ROI for bone morphometric analysis was defined in the first scan and transferred to the second and third scan. While the ROI was equal for the follow-up scans of the same animal, it was not possible to use the same ROI for different animals due to differences in the original animal positioning. Therefore, changes in bone morphometric parameters over time were compared between groups instead of the parameters themselves. Evaluation included the changes in BV, tr.th. and BD.

\section{Post-mortem evaluation of articular cartilage}

After the third $\mu \mathrm{CT}$ scan, animals were euthanised using a pentobarbital overdose. Animals were positioned in right-lateral recumbency and pentobarbital was injected intracardially $(800-900 \mathrm{mg} /$ animal, Narkodorm ${ }^{\circledR}$, CP-Pharma) while being deeply anaesthetised.

Tissue was carefully removed and the knee joint exposed. In situ status of the cartilage was documented. Evaluation of cartilaginous changes was performed according to Laverty et al. (2010) including cartilage changes and the presence of osteophytes. Values for cartilage changes ranged from 0 (surface smooth with normal cartilage) up to 4 (complete 
cartilage erosion with subchondral bone exposed). Values for occurrence of osteophytes ranged from 0 (absent) to 3 (severe). Exact scores are displayed in Table 2.

\section{Histological evaluation}

After cartilage documentation, bone samples were harvested. The distal parts of right and left femora were processed for histological evaluation. Both condyles were fixed each in $4 \%$ neutral buffered formalin for a minimum of 1 week. After successful fixation, medial and lateral condyles were separated using a band saw (300 CL, Exakt, Norderstedt, Germany) and embedded in Technovit 9100 following the manufacturer's embedding protocols (Kulzer GmbH, Hanau, Germany). Approximately $5 \mu \mathrm{m}$ sections were prepared using a hard tissue rotation microtome (Leica RM2255, Nussloch, Germany). Slides were stained with safranin-O-fast green. Then, safranin-O-stained slides were evaluated using a semiquantitative score according to Laverty et al. (2010) that included the parameters staining (0-6), structure (0-11), chondrocyte density (0-4) and cluster formation (0-3).

\section{Element imaging}

The element analysis was performed using a Tornado M4 $\mu$ XRF spectrophotometer (Bruker Nano, Berlin, Germany) as previously described (Helmholz et al., 2019). The X-ray spectrometer was equipped with a $\mathrm{Rh}$ tube working at a voltage of $50 \mathrm{kV}$ and an anode current of $600 \mu \mathrm{A}$. The sample chamber was operated under vacuum conditions ( $2 \mathrm{kPa}$ ). The methacrylate embedded explants were analysed by elemental imaging leading to elemental maps 12 weeks after the second surgery. The material was mapped with a spot size of $25 \mu \mathrm{m}$, a spot distance of $15 \mu \mathrm{m}$ and an acquisition time of $1.5 \mathrm{~ms} /$ pixel. Radiation was detected using a Bruker XFlash ${ }^{\circledR}$ Silicon Drift Detector $30 \mathrm{~mm}^{2}$, energy resolution $<145 \mathrm{eV}$ for
250,000 counts/s. The object of interest was selected manually and element quantification was done by averaging the intensity signals over the object map area. Background correction was achieved by subtracting the average peak intensities obtained from a pure methacrylate region. Data evaluation and image processing were performed using the Bruker ESPRIT microanalysis software version 1.3.0.3273.

\section{Statistics}

Statistical analysis was performed using SPSS 10.0. Results were tested for normal distribution. Either ANOVA or Kruskal-Wallis and Mann-Whitney-U Test were used. $p \leq 0.5$ was considered statistically significant.

\section{Results}

\section{Microstructure of the $\mathrm{Mg}$ cylinders}

Fig. 2 shows the microstructure of the cylinders. Grain size and shape were independent of the transversal or longitudinal direction, with globular grains. The micrographs of the transverse direction (Fig. 2a) and the longitudinal direction (Fig. 2b) showed a larger number of twins in the cutting direction, which was inverse to the punching direction. The deformation twins can be recognised by the cigar shape in the grains. These deformation twins are caused by the low plasticity of $\mathrm{Mg}$ crystals as a deformation mechanism in the grains. These are macroscopically visible crystal defects that have a mirror-symmetrical crystal structure across the twin boundary. The number of deformed grains and twins increased in the longitudinal direction (Fig. 2b) near the sides where the punching process cut the wire into the cylinders. No grains or twins were visible directly on the cutting edge (clearly visible in Fig. $2 \mathbf{b}$ on the right).
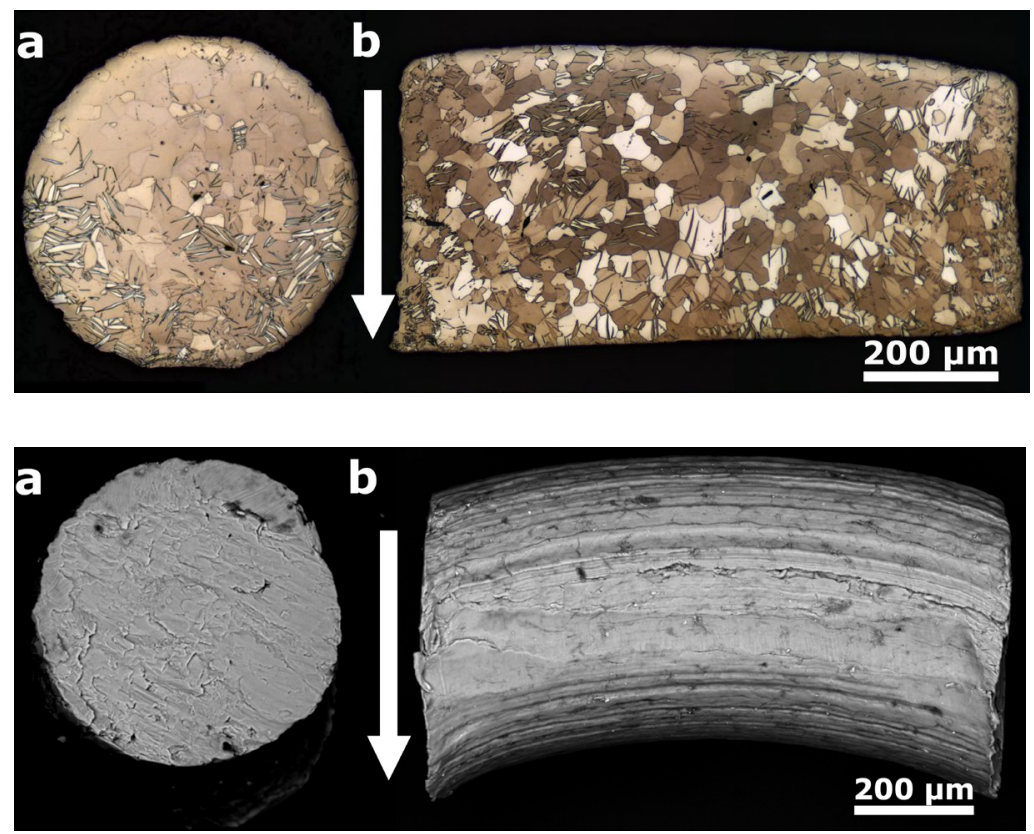

Fig. 2. Micrographs of (a) transversal and (b) longitudinal direction of the Mg cylinder. The arrow indicates the process direction of cutting, which was inverse to the punching direction. The scale bar is valid for both micrographs.

Fig. 3. SEM micrographs of the $\mathrm{Mg}$ cylinder from (a) the cutting surface and (b) the drawing surface. The arrow indicates the process direction of cutting, which was inverse to the punching direction. The scale bar is valid for both micrographs. 


\section{Shape}

Fig. 3a shows the smooth surface of the main part following the punching process, with small scratches resulting from the removal of the punch. The arrow indicates the process direction for the punching. Fig. $3 \mathbf{b}$ shows the bending of the cylinder caused by the punching process.

\section{Immersion test}

The results of the immersion test by the weight-loss method, performed at 3, 7, 14, 21 and $28 \mathrm{~d}$ under both normoxia and hypoxia, are presented in Fig. 4. The degradation rate was found to be slightly lower under hypoxia conditions when compared to normoxia conditions, as revealed by the slope value from applying the linear regression fit equation. The morphology after degradation changes is depicted

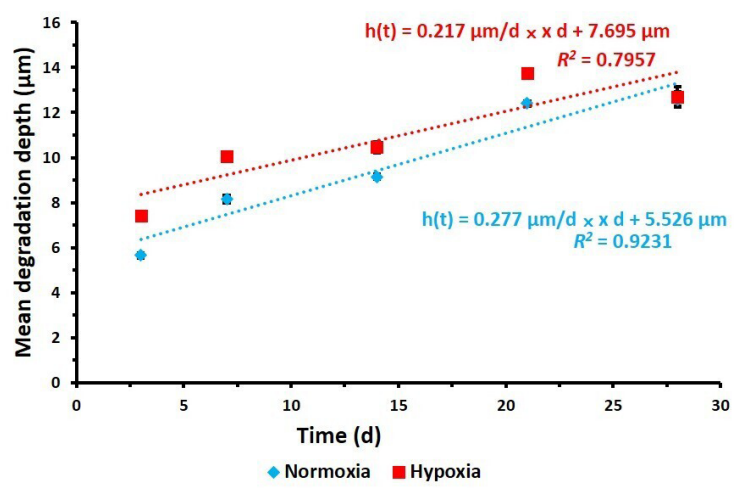

Fig. 4. Mean degradation depth $(h ; \mu m)$ as per weight-loss method for $\mathrm{Mg}$ cylinders under normoxia and hypoxia. in the optical microscopy images of the surface morphology (Fig. 5). The Mg mean degradation depth over time was in the order of the $\mu \mathrm{m}$. This linear mathematic description of degradation was described by Nidadavolu et al. (2016). Under hypoxia, the initial loss was higher compared to normoxia, with higher nominal values up to $28 \mathrm{~d}$ of immersion. However, the degradation under normoxia, with $0.277 \mu \mathrm{m} / \mathrm{d}$ (0.102 $\mathrm{mm} /$ year), showed a stronger increase over time compared to the hypoxia, with $0.217 \mu \mathrm{m} / \mathrm{d}$ $(0.080 \mathrm{~mm} /$ year $)$ in the time interval of $3-28 \mathrm{~d}$. The initial degradation was too fast and difficult to detect or interpret, as also explained by Nidadavolu et al. (2016), but the lower $\mathrm{O}_{2}$ content showed a clear effect on the initial degradation rate. In the longterm immersion test carried out for 6 and 8 weeks, no significant differences were measurable. After 8 weeks, only traces of remaining particles could be observed and the measurement of weight loss, needed to quantify the degradation, was impossible. The $\mathrm{pH}$ values measured, which ranged between 7 and 9, are listed in Table 3. The osmolality (Table 3) values were in the same range as the control. The percentage of $\mathrm{Mg}$ element concentration release into the medium measured through AAS (Table 3) revealed a larger loss in normoxia than in hypoxia. The treatment of $\mathrm{Mg}$ cylinder with the self-made insertion instrument, as used for the in vivo placement of the implants, did not significantly influence the in vitro degradation behaviour.

\section{Clinical assessment}

Following both surgeries, none of the rabbits showed an impairment in general condition and behaviour

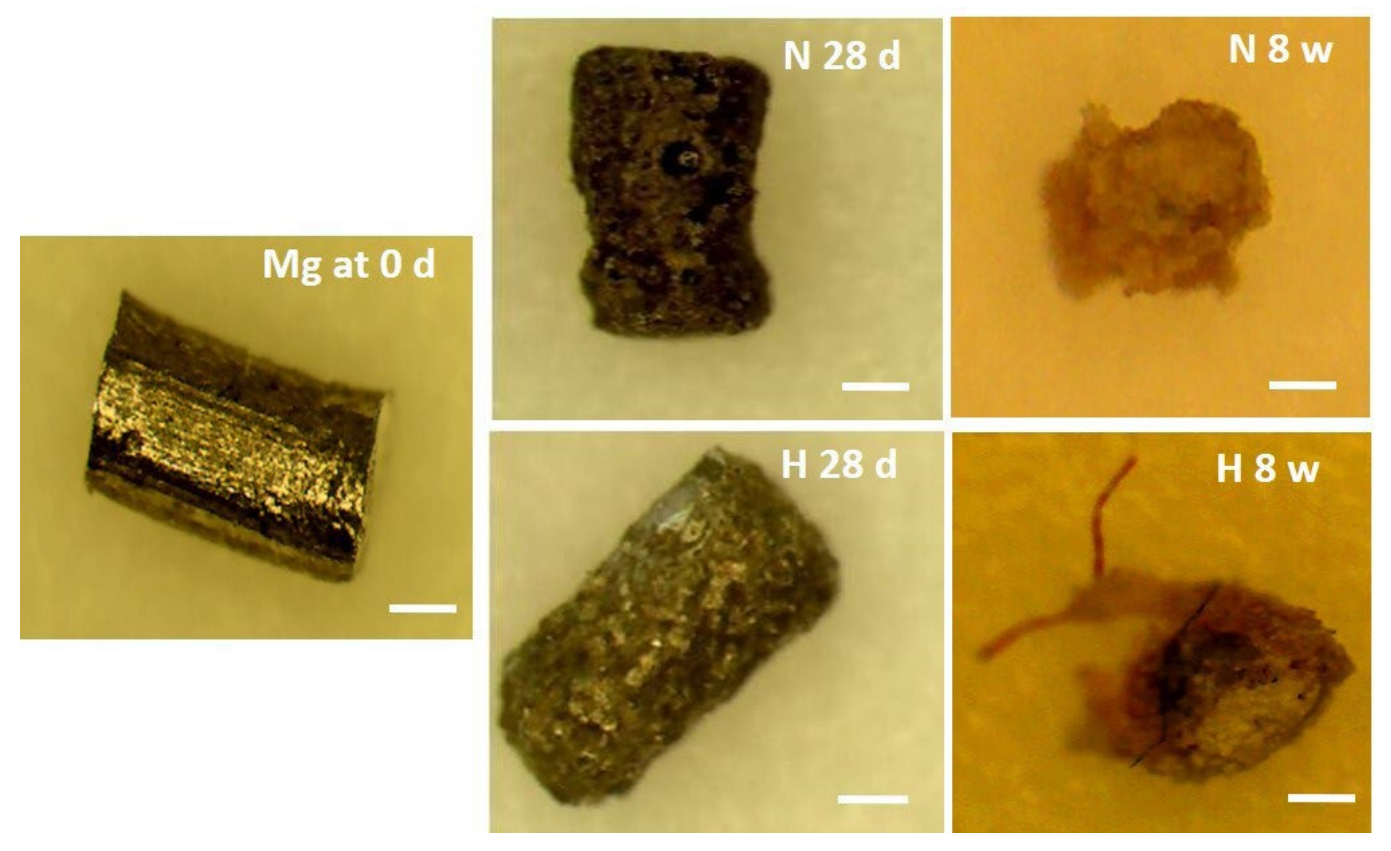

Fig. 5. Qualitative representation of surface morphology of degraded cylinders after chromic acid treatment. The image on the left represents a cylinder at day 0 (before chromic acid treatment), while top images represent samples at normoxia $(\mathrm{N})$ and bottom at hypoxia $(\mathrm{H})$ after $28 \mathrm{~d}$ and 8 weeks $(\mathrm{W})$, respectively. Scale bar: $100 \mu \mathrm{m}$. 
Table 3. $\mathrm{pH}$, osmolality changes and percentage Mg loss measured using AAS following 3, 7, 14, 21 and $28 \mathrm{~d}$ of immersion test. Mean \pm standard deviation; $n=6$.

\begin{tabular}{|c|c|c|c|c|c|c|}
\hline \multicolumn{2}{|c|}{} & \multicolumn{5}{|c|}{ Time (d) } \\
\cline { 3 - 7 } \multicolumn{2}{|c|}{} & $\mathbf{3}$ & $\mathbf{7}$ & $\mathbf{1 4}$ & $\mathbf{2 1}$ & $\mathbf{2 8}$ \\
\hline \multirow{3}{*}{$\mathbf{p H}$ control } & Normoxia & $7.21 \pm 0.01$ & $7.57 \pm 0.02$ & $7.77 \pm 0.04$ & $8.12 \pm 0.05$ & $8.25 \pm 0.01$ \\
\cline { 2 - 7 } & Hypoxia & $7.59 \pm 0.00$ & $7.68 \pm 0.01$ & $7.99 \pm 0.00$ & $8.01 \pm 0.00$ & $8.15 \pm 0.02$ \\
\hline \multirow{2}{*}{$\mathbf{p H}$} & Normoxia & $8.13 \pm 0.07$ & $8.14 \pm 0.02$ & $8.17 \pm 0.10$ & $8.26 \pm 0.10$ & $8.59 \pm 0.12$ \\
\cline { 2 - 7 } & Hypoxia & $8.11 \pm 0.02$ & $8.25 \pm 0.00$ & $8.29 \pm 0.03$ & $8.38 \pm 0.14$ & $8.68 \pm 0.09$ \\
\hline $\begin{array}{c}\text { Osmolality control } \\
\text { (osmol/kg) }\end{array}$ & Normoxia & $0.32 \pm 0.00$ & $0.33 \pm 0.01$ & $0.39 \pm 0.00$ & $0.39 \pm 0.01$ & $0.42 \pm 0.02$ \\
\cline { 2 - 7 } & Hypoxia & $0.33 \pm 0.02$ & $0.34 \pm 0.01$ & $0.36 \pm 0.03$ & $0.38 \pm 0.00$ & $0.41 \pm 0.01$ \\
\hline $\begin{array}{c}\text { Osmolality } \\
\text { (osmol/kg) }\end{array}$ & Normoxia & $0.40 \pm 0.00$ & $0.50 \pm 0.01$ & $0.48 \pm 0.01$ & $0.41 \pm 0.03$ & $0.439 \pm 0.01$ \\
\cline { 2 - 7 } & Hypoxia & $0.42 \pm 0.01$ & $0.43 \pm 0.04$ & $0.45 \pm 0.00$ & $0.46 \pm 0.01$ & $0.43 \pm 0.01$ \\
\hline \multirow{2}{*}{$\mathbf{C ~ M g ~ ( m m o l / L ) ~}$} & Normoxia & $0.86 \pm 0.13$ & $1.74 \pm 0.25$ & $2.51 \pm 0.13$ & $3.14 \pm 0.69$ & $3.68 \pm 0.13$ \\
\cline { 2 - 7 } & Hypoxia & $0.59 \pm 0.06$ & $1.24 \pm 0.04$ & $1.72 \pm 0.08$ & $2.24 \pm 0.07$ & $2.86 \pm 0.38$ \\
\hline \multirow{2}{*}{\begin{tabular}{c} 
Mg loss (\%) \\
\cline { 2 - 7 }
\end{tabular}} & Normoxia & 8.70 & 17.52 & 24.92 & 31.51 & 36.66 \\
\cline { 2 - 7 } & Hypoxia & 5.06 & 10.79 & 17.20 & 22.45 & 28.39 \\
\hline
\end{tabular}

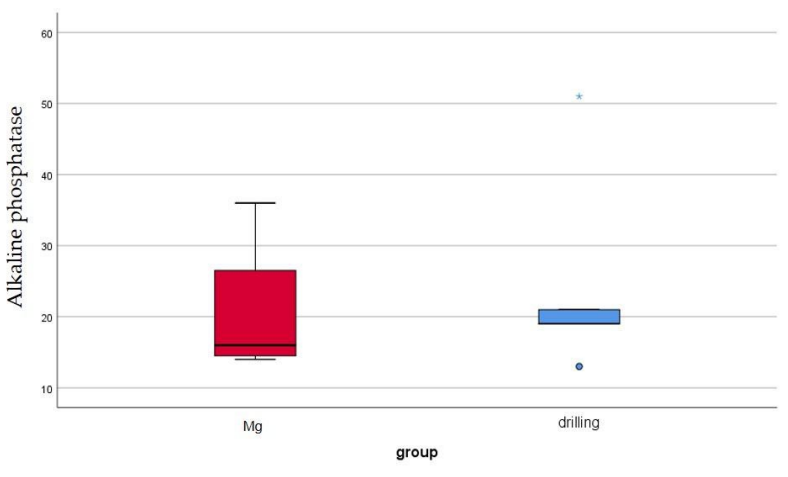

Fig. 6. AP values of blood samples taken 8 weeks after either implantation of $\mathrm{Mg}$ cylinders or drilling of holes only. Values in $\mathrm{U} / \mathrm{L}$, normal range 9.1-94.7 U/L. Blue dot: outlier with $>1.5$ and $<3.0$ of the interquartile range. Blue asterisk: outlier with $>3.0$ of the interquartile range.

when recovering from the anaesthesia. After ACLT, the median weight reduction appeared to be higher in animals that received $\mathrm{Mg}$. However, this difference was not statistically significant.

Regarding the clinical scoring, including the parameters of wound swelling and redness as well as closure of suture, no significant changes could be seen between both groups. Corresponding to the severity of the surgical procedure, scores were higher after the second intervention. However, also after the second surgery, most animals showed no increased clinical parameters after $14 \mathrm{~d}$. As a reaction to the opening and closing of the joint capsule, all animals developed sturdy protuberances in the region of the medial femoral condyle. These protuberances ranged from slight surface irregularities up to distinct, firm excesses, all with no sensitivity to pain.

Regarding passive pain, only a mild increase could be observed for both groups after both surgeries, with slightly higher values after the second surgery. This trend could also be observed for the active pain evaluation. After the first surgery, most animals presented moderate abnormalities in weight loading of the limb during motion but also partly during resting. This was represented by a slight lifting of the limb and shifting of weight during sitting. 2 weeks after surgery these changes were only slight in all animals. Normal weight loading was regained on day 66 in all animals. After the second surgery, symptoms of moderate lameness were apparent for a longer period (up to day 7) - but afterwards all animals recovered faster so that already after $14 \mathrm{~d}$ most rabbits only showed mild symptoms. Normal weight loading after the second surgery in all animals was achieved on the $48^{\text {th }}$ postoperative day and therewith 2.5 weeks earlier than after ACLT.

\section{Blood samples}

AP values of all evaluated blood samples were within the normal range. Although the difference in $\mathrm{AP}$ values between the $\mathrm{Mg}$ group and the drilling group was not statistically significant, animals with implanted $\mathrm{Mg}$ showed lower values compared to animals without $\mathrm{Mg}$ (Fig. 6).

\section{$\mu \mathrm{CT}$}

3 animals in the $\mathrm{Mg}$ group and 4 animals in the drilling group were included in $\mu \mathrm{CT}$ analysis. The other animals $(\operatorname{Mg} n=2$, drilling $n=1)$ had to be excluded due to positioning errors or scan artefacts that did not allow for correct contouring and evaluation. $\mu \mathrm{CT}$ results showed distinct differences between both groups. However, changes were more distinct in the femur, where significant differences could be found for two of three parameters.

Regarding BV and tr.th., both groups showed a clear increase in both parameters for the femur (Fig. 
7). Percentage increase in the $\mathrm{Mg}$ group from scan 1 (drilling/implantation) to scan 2 (4 weeks after surgery) was $29.4 \%$ for BV and $29.1 \%$ for tr.th. From scan 2 to scan 3 (8 weeks after surgery), the further increase for both parameters was $11.6 \%$ and $18.7 \%$, respectively, summing up to a total increase in BV from scan 1 to scan 3 of $44.5 \%$ and a total increase in tr.th. of $53.2 \%$ in the $\mathrm{Mg}$ group. In comparison, increase in $\mathrm{BV}$ in the drilling group from scan 1 to scan 3, with $15.1 \%$ (scan 1-scan 2: $8.1 \%$; scan 2-scan 3: $6.4 \%$ ), and tr.th., with $16.9 \%$ (scan 1-scan 2: $8.3 \%$; scan 2-scan 3: $7.7 \%$ ), was significantly lower than in the $\mathrm{Mg}$ group, where the increase was nearly 3 times as high $\left(p_{B V}=0.025, p_{t r \text { th }}=0.025\right.$; Fig. 7).

Changes of BV and tr.th. in the tibia showed the same tendency as in the femur, however at a lower level (Fig. 7). In the Mg group, from scan 1 to scan 3,
BV increased by $27.2 \%$ (scan 1 to scan 2: $12.8 \%$; scan 2 to scan 3: $14.9 \%$ ), while tr.th. increased by $27.9 \%$ (scan 1 to scan 2: $12.8 \%$; scan 2 to scan 3: $13.4 \%$ ). Changes in the drilling group were smaller than in the $\mathrm{Mg}$ group. In total, BV increased by $12.6 \%$ (scan 1 to scan 2: $8.1 \%$; scan 2 to scan 3: $4.5 \%$ ) and tr.th. by $18.4 \%$ (scan 1 to scan 2: $11.1 \%$; scan 2 to scan 3: $7.9 \%$ ). Therewith, BV in the Mg group was more than twice as high as in the drilling group.

Differences between both groups were smaller regarding the $\mathrm{BD}$. In the femur, $\mathrm{BD}$ increased in both groups from scan 1 (implantation of cylinders/ drilling only) to scan 3 (end of follow up period, 8 weeks later). Noticeably, the dynamic was different in both groups. While there was nearly no change in density from scan 1 to scan 2 in the $\mathrm{Mg}$ group $(-0.2 \%)$, animals of the drilling group showed a

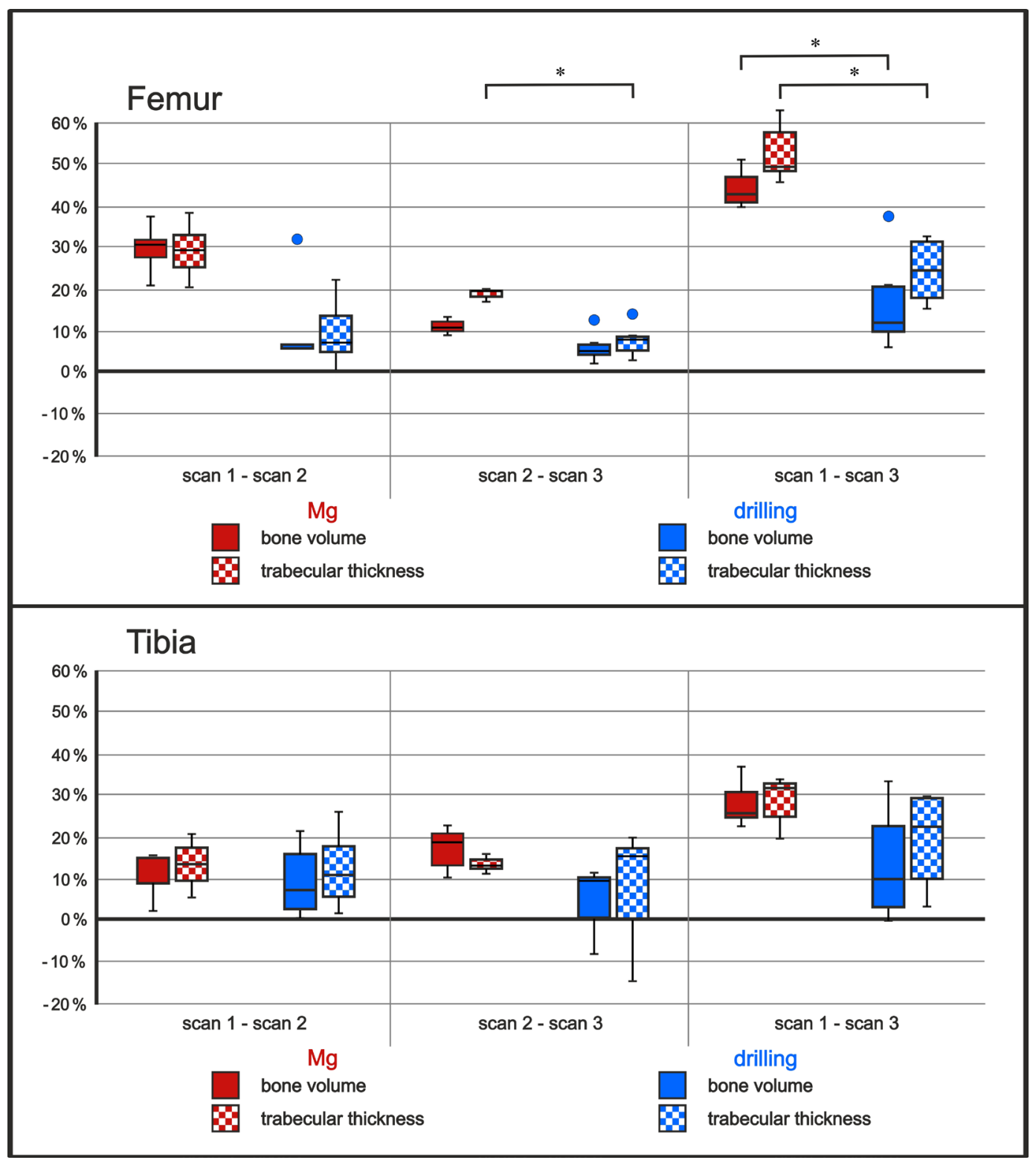

Fig. 7. Boxplots of $\mu \mathrm{CT}$ evaluation for BV and tr.th. of both groups [Mg (red) and drilling (blue)] in the femur (upper part) and tibia (lower part). Values display the percentage changes of the measured parameter in the indicated time span ( $\Delta$ scan $x /$ scan y). Scan 1: drilling/drilling and insertion of Mg cylinders. Scan 2: 4 weeks after drilling/insertion of Mg. Scan 3: 8 weeks after drilling/insertion of Mg. Black asterisk: significant differences between the indicated groups, $p<0.05$. Dots: outlier with $>1.5$ and $<3.0$ of the interquartile range. 
slight increase of $1.3 \%$. In the following weeks, BD in the $\mathrm{Mg}$ group increased by $1.8 \%$, which resulted in an overall increase from scan 1 to scan 3 of $1.5 \%$. BD in the drilling group also slightly increased further from scan 2 to scan 3 (1.1\%), which summed up to a total increase in BD from scan 1 to scan 3 of $2.5 \%$. Overall, BD of the drilling group appeared to increase slightly more than in the $\mathrm{Mg}$ group. However, all changes were small and differences not statistically significant. All values are depicted in Fig. 8. BD in the tibia showed a clearly different progress, which was similar for both groups. While BD decreased slightly but clearly from scan 1 to scan 2 (Mg: $-2.1 \%$; drilling: $-1.5 \%$ ), there was an equally slight but clear increase from scan 2 to scan 3 (Mg: $1.8 \%$; drilling: $1.2 \%$ ), summing up to a nearly unchanged BD when considering the 8 weeks period from scan 1 to scan 3 (Mg: - 0.4; drilling: $-0.3 \%$, Fig. 8).

\section{Post-mortem evaluation of articular cartilage}

Cartilage lesions were found in both tibial and femoral articular surfaces in all joints. In the $\mathrm{Mg}$ group, lesions were more severe in the tibia than in the femur, represented by a significantly higher score $(p=0.41)$ (Fig. 9). In the drilling group, the tendency was the opposite: cartilage lesions appeared to be less severe in the tibia. However, this difference was not statistically significant. Comparing the cartilage lesions in the femur between $\mathrm{Mg}$ group and drilling group, all differences were also not statistically significant. The Mg group showed a clear tendency to less severe changes in the femur, while lesions in the tibia of the $\mathrm{Mg}$ group were more pronounced. Osteophytes were evaluated at the femur and they appeared to be slightly more severe in rabbits of the Mg group than in those of the drilling group. Again, this result was not statistically significant (Fig. 9).

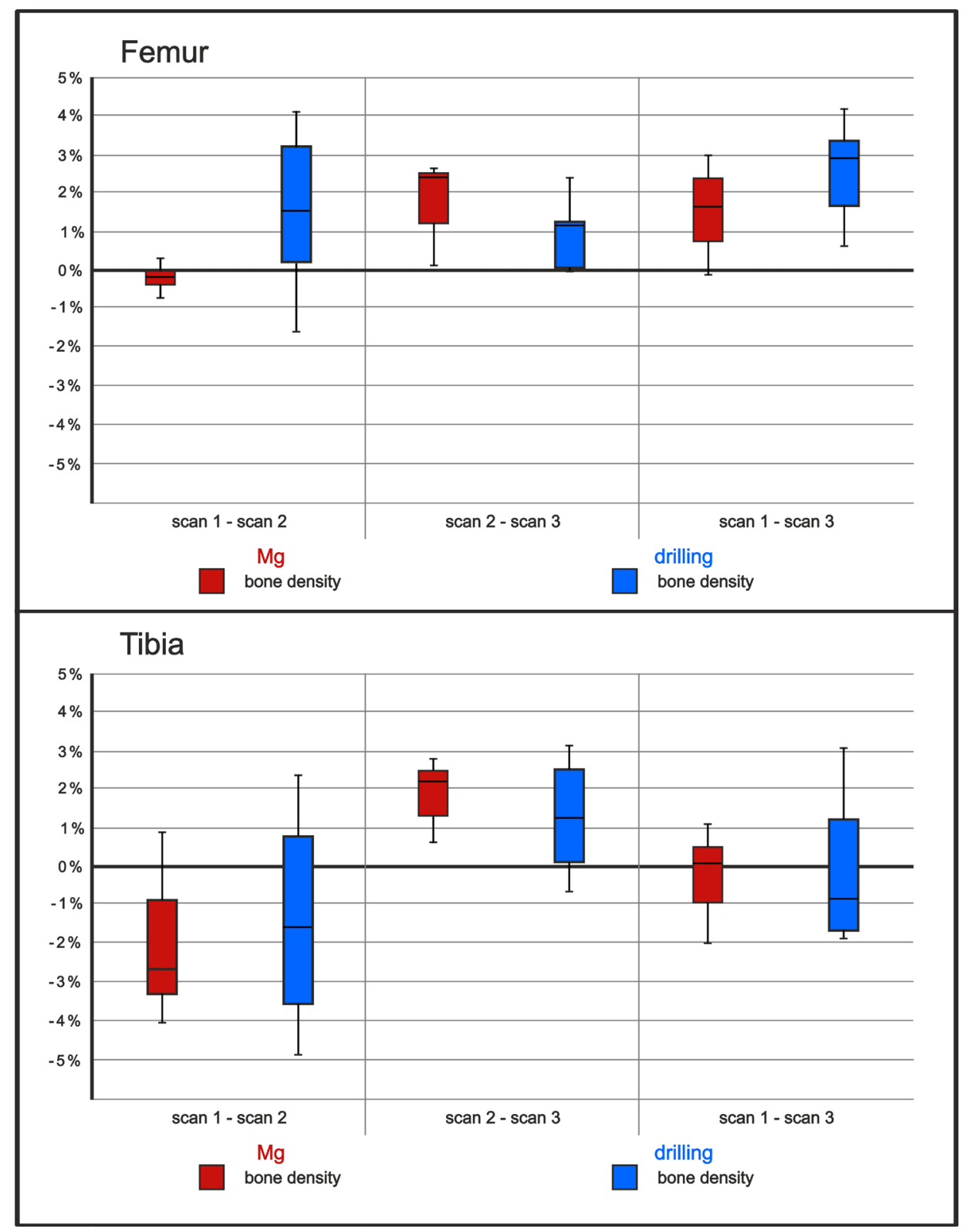

Fig. 8. Boxplots of $\mu \mathrm{CT}$ evaluation for BD of both groups [Mg (red) and drilling (blue)] in the femur (upper part) and tibia (lower part). Values display the percentage changes of the BD calculated for the indicated time span ( $\Delta$ scan $x /$ scan y). Scan 1: drilling/drilling and insertion of Mg. Scan 2: 4 weeks after drilling/insertion of Mg. Scan 3: 8 weeks after drilling/insertion of Mg. 


\section{Histological evaluation}

As seen also in the macroscopical evaluation of articular cartilage, safranin $\mathrm{O} /$ fast-green-stained slices showed different stages of cartilage lesions in all samples (Fig. 10). In both groups, nearly unchanged cartilage was found (Fig. 9a,d, $, \mathbf{g}, \mathbf{h}$ ) as well as clearly progressed cartilage lesions, represented by areas not stained after performing safranin $\mathrm{O}$-fast green staining (Fig. 10b,c,e,i). Also, total cartilage destruction up to the SBP occurred (Fig. 10f). However, evaluation by scoring could not reveal any difference between samples with implanted $\mathrm{Mg}$ cylinders and samples with drilling holes, which was measurable only when scores of medial and lateral condyles were summed to a total score (Fig. 11). In the $\mathrm{Mg}$ group, left, non-operated control samples

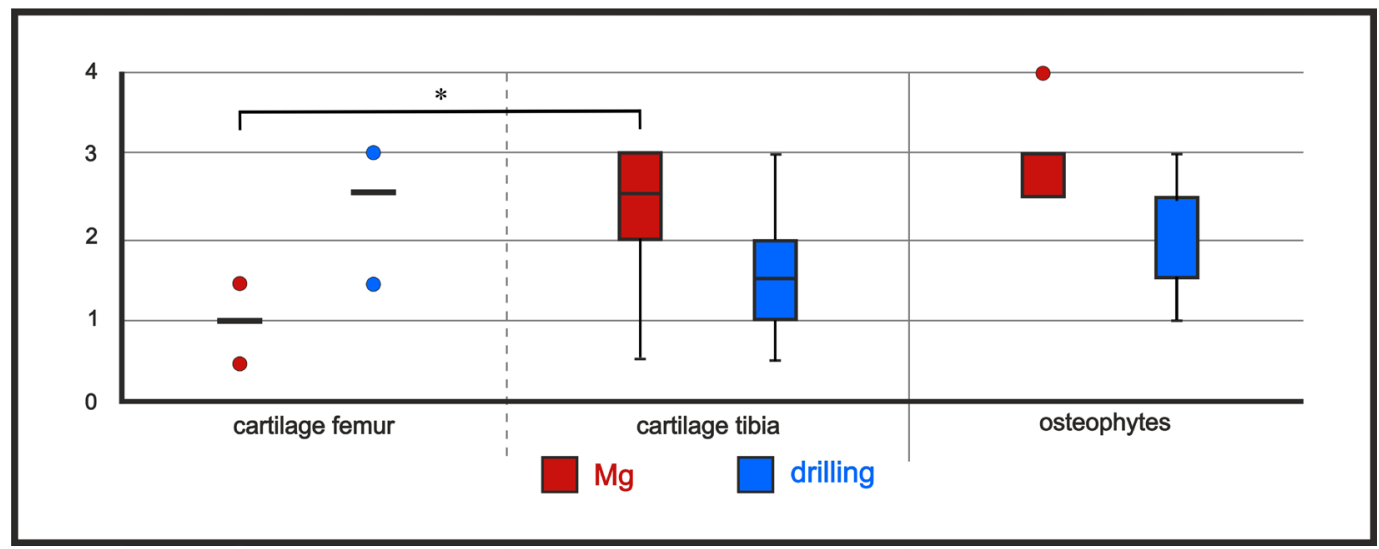

Fig. 9. Macroscopical evaluation of articular cartilage by score values according to Laverty et al. (2010). Black asterisk: significant differences between the indicated groups, $p<0.05$. Red and blue dots: outlier with $>1.5$ and $<3.0$ of the interquartile range.

right, operated side - Mg-beads
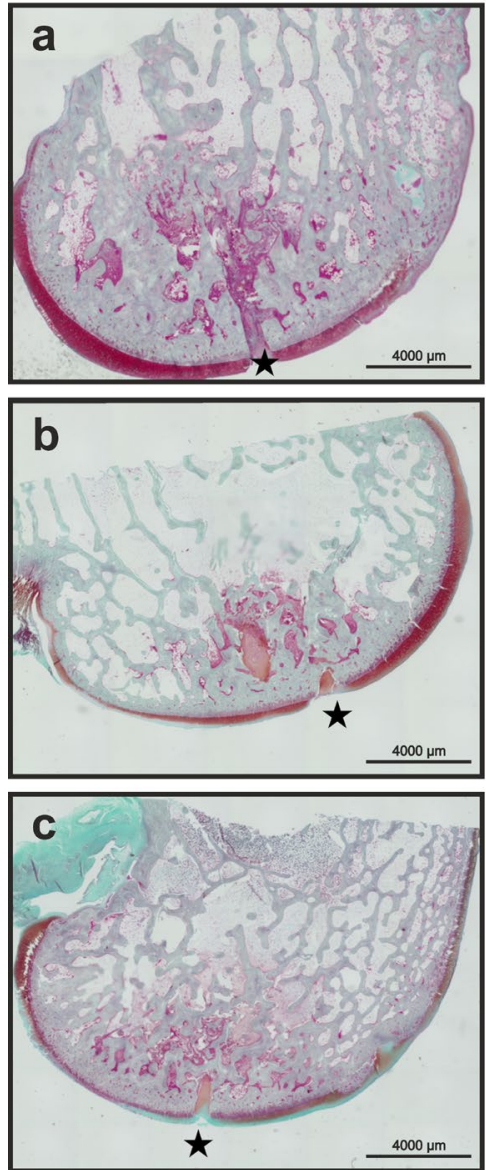

right, operated side - drilling
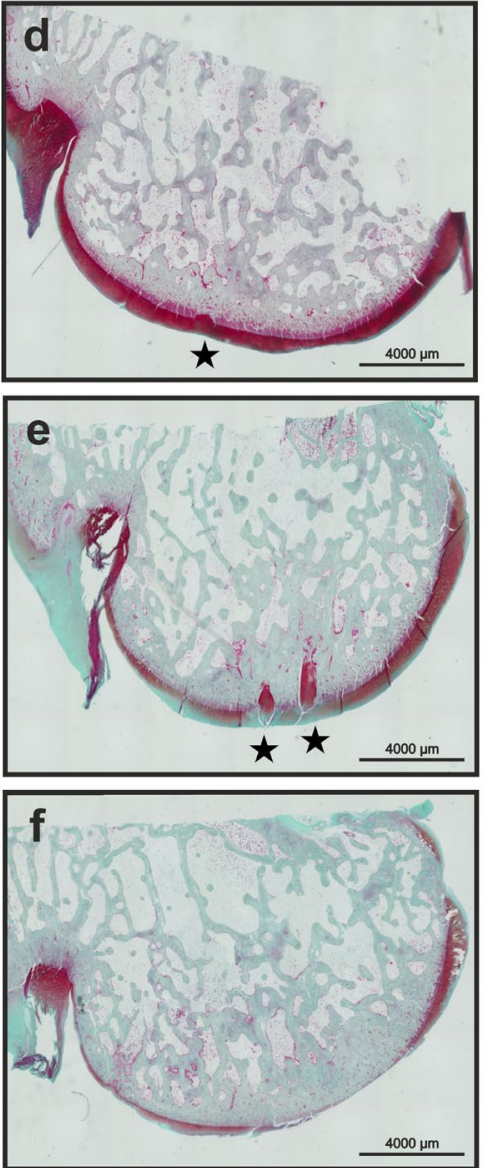

\section{left, non-operated side - both}
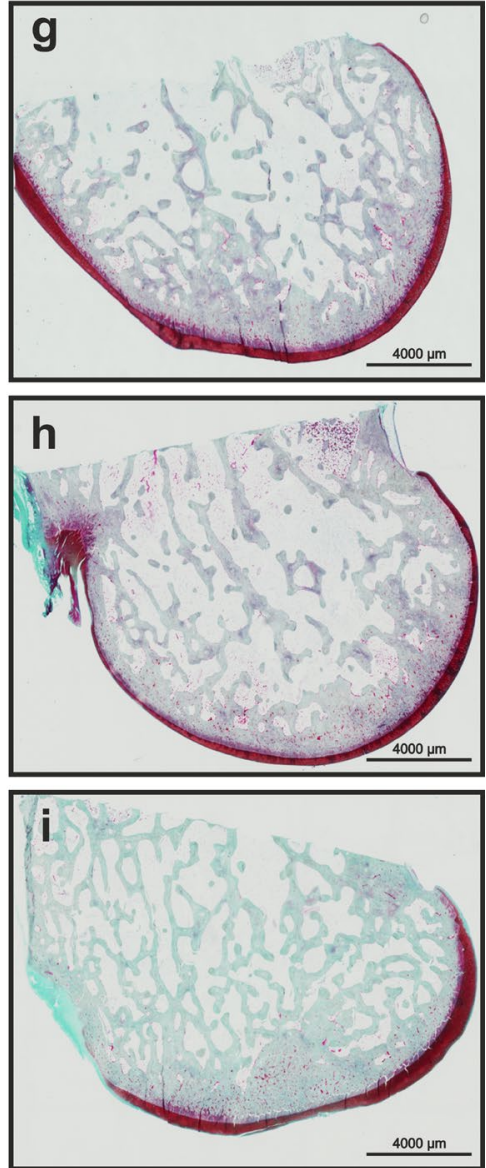

Fig. 10. Representative images of histological, safranin-O/fast green-stained slices for all groups. (a-c) Samples of right, operated femora with implanted Mg. (d,f) Samples of right, operated femora with drilling holes only. (g,h) Samples of left, non-operated femora; (g) Mg group, (h,f) drilling group. * transverse section of drill hole. 
showed significantly fewer changes, represented by lower score values. In the drilling group, the same relation between left and right cartilage could be found, even though in this group the difference was not statistically significant. Regarding the left control samples of animals with drilling only, they showed significantly stronger lesions than samples of the $\mathrm{Mg}$ group (Fig. 11).

If medial and lateral condyles were regarded separately, the same tendency could be shown as for the summed scores. However, medial condyles showed slightly increased changes in the $\mathrm{Mg}$ group compared to the drilling group, while the situation was the opposite for the lateral condyles. Noticeably, changes in the medial condyles of the drilling group were more severe in the left control than in the operated right samples.

\section{Element imaging}

Elemental imaging of the explants did not show any remaining traces of $\mathrm{Mg}$ in the bone cortex (Fig. 12). This reflected the complete degradation of the cylinders within 12 weeks. Another aspect of the medical application of degradable metallic implants is a potential tissue enrichment in elemental components. Therefore, a full scan of the ex vivo specimen was performed to detect variances in the elemental distribution. In Fig. 12, Ca and P represent the mineralised tissue and $S$ a proteinaceous tissue. The element content of S, P and Ca did not show significant differences between the $\mathrm{Mg}$ group and the drilling group. Traces of $\mathrm{Mg}$ were detectable at the lower limit of detection. There appeared to be a slightly increased level in the Mg-treated specimen in relation to the control side and the drilling group. However, this trend was not significant. The K level showed a significant increase comparing the $\mathrm{Mg}$ side to the drilling side (Fig. 13).

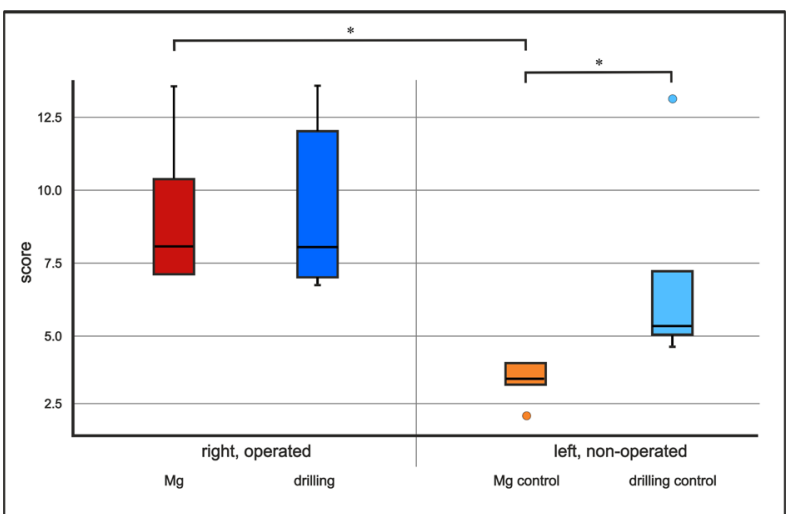

Fig. 11. Results of histological evaluation of safranin $\mathrm{O} /$ fast greensstained slices displayed as boxplots. Values represented were scored according to Laverty et al. (2010). Samples prepared from right, operated knees showed higher score values than samples prepared from left, non-operated bones. Black asterisk: significant differences between the indicated groups with $p<0.05$. Orange and light blue dots: outlier with $>1.5$ and $<3.0$ of the interquartile range.

\section{Discussion}

The influence of small, implanted Mg cylinders on the subchondral bone quality was examined in comparison to solely drilling holes in an OA rabbit model. 12 weeks after initiating OA by ACLT and incision of the medial meniscus, 5 rabbits received either pure $\mathrm{Mg}$ cylinders or the same quantity of drilling canals into lateral and medial compartments of both, tibial and femoral articular bone parts and were studied during an 8-week follow-up period.

The material processing ensured the homogeneity of the applied cylinders with comparable degradation behaviour. The examined globular grain structure without elongated grains showed that the recrystallisation treatment was sufficient to produce a completely recrystallised microstructure. The results of the microstructure and SEM images were in good agreement and showed the expected macro and micro results from the punching process. The bending was an effect of the stress during the cutting process, which led to plastic deformation of the wire and finally the cylinders. Therefore, the shape was not a perfect cylinder. At the same time, this deflection caused fewer twins on the stretched side than on the opposite side, which was due to the anisotropic behaviour and the low plasticity of the Mg crystal. On the compressed side, twinning required less critical resolved shear stress than on the stretched side (Alaneme and Okotete, 2017; Dobroň et al., 2011). On and near the cutting planes, the microstructural grain structure was strongly deformed, so that grains or twins could not be recognised. This was due to the high shear force caused by the punching process, with $100 \%$ plastic deformation. The drawing process caused the relief of the cylinders, especially by the drawing die in the last drawing step.

The in vitro degradation of the small implants was relatively fast compared to other implant geometries, which might be due to their larger surface area (Nidadavolu et al., 2016). Taking into account the reduced oxygen level in a tissue, the in vitro degradation was tested under hypoxic conditions of $3 \% \mathrm{O}_{2}$ to mimic the in vivo conditions. Articular cartilage is a tissue with no direct blood supply and the oxygen level ranges from $10 \%$ to less than $1 \%$. Furthermore, the cellular oxygen partial pressure is below $2.5 \%$ (Carreau et al., 2011; Coyle et al., 2009). The degradation of Mg implants in hypoxic conditions slightly increased over time after a stronger initial degradation. During degradation, $\mathrm{Mg}$ reacted with the $\mathrm{O}_{2}$ dissolved in the medium and with $\mathrm{H}_{2} \mathrm{O}$ to form an initial $\mathrm{MgO}$ layer at the interface. For hypoxic conditions, this effect could be reduced due to the lower partial $\mathrm{O}_{2}$ pressure in the medium. The formation of the protective layer by the degradation products was reduced and the initial weight loss was greater compared to the normoxic condition. The different oxygen regimes might be one explanation why in vitro measured degradation rates were faster than in vivo (Martinez Sanchez et 
al., 2015). Therefore, an adaptation of the in vitro degradation test conditions could be recommended when considering the in vivo parameters. However, under both oxygen conditions, only traces of $\mathrm{Mg}$ cylinders could be found after 8 weeks in vitro and there were no $\mathrm{Mg}$ particle traces detectable in the drill holes as observed by elemental imaging.

Systemic effects of $\mathrm{Mg}$ on measured parameters such as AP or general behaviour of rabbits were not observed, whereas local effects were significantly different between $\mathrm{Mg}$ and drilling group, although group sizes for statistical analysis were low. Results of the three $\mu \mathrm{CT}$ scans showed that the implanted $\mathrm{Mg}$ significantly influenced the bone remodelling in the SBP and underlying trabeculae. BV and tr.th. of the femoral condyles were significantly higher in rabbits with implanted $\mathrm{Mg}$ cylinders than in those with drilling holes. The same tendency appeared to be present in the tibia, but without statistical significance. Higher BV and tr.th. in bones of OA joints was already reported (Bobinac et al., 2013). In the present study,
OA was similar in both groups and differences in the further progress after second surgery was caused by different treatments. Osteoconductivity is one of the predominantly reported properties of implanted Mg-based materials in orthopaedic research (Kraus et al., 2012; Willbold et al., 2017; Witte et al., 2005). Also, Yu et al. (2019) showed an increase in BV after the implantation of a polylactide-coglycolide/ tricalcium phosphate composite scaffold that was additionally supplemented by a $\mathrm{Mg}$ powder. The authors discussed that the release of $\mathrm{Mg}$ ions led to an increased $\mathrm{pH}$ value in the scaffold region, which enhanced the osteoblastic activity (Shen et al., 2012; Yu et al., 2019). The results of the present study showed that the reported osteoconductive effect of $\mathrm{Mg}$ implants were also performed by the small sized cylinders and therewith reached the desired goal to affect the SBP. The osteoconductive influence during the first 4 weeks was more pronounced than in the following 4 weeks. The significant influence in the femur compared to the less pronounced effect in

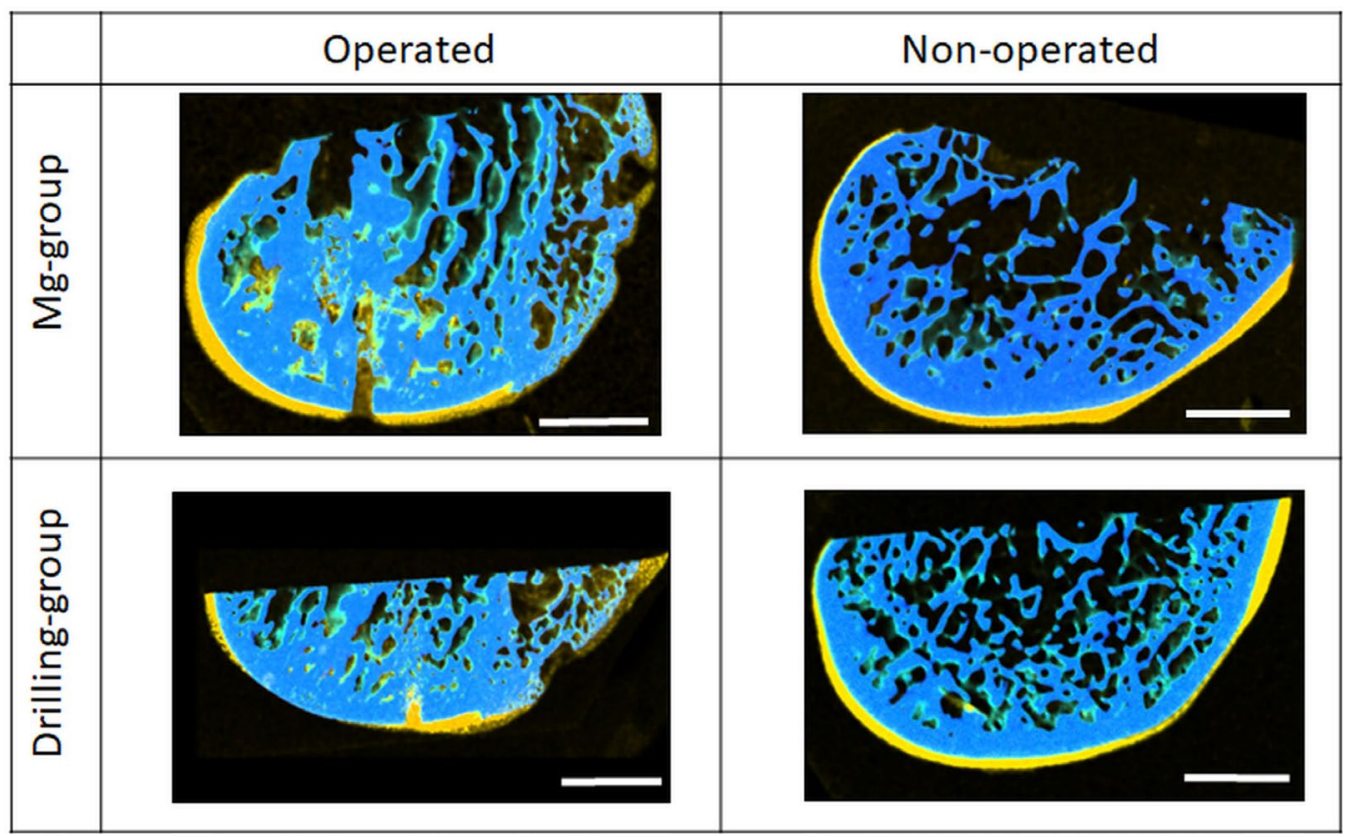

Fig. 12. Elemental maps of Mg-group and drilling group, operated and non-operated side. Ca: light blue; P: dark blue; S: yellow; K: orange. Scale bar: $3 \mathrm{~mm}$.

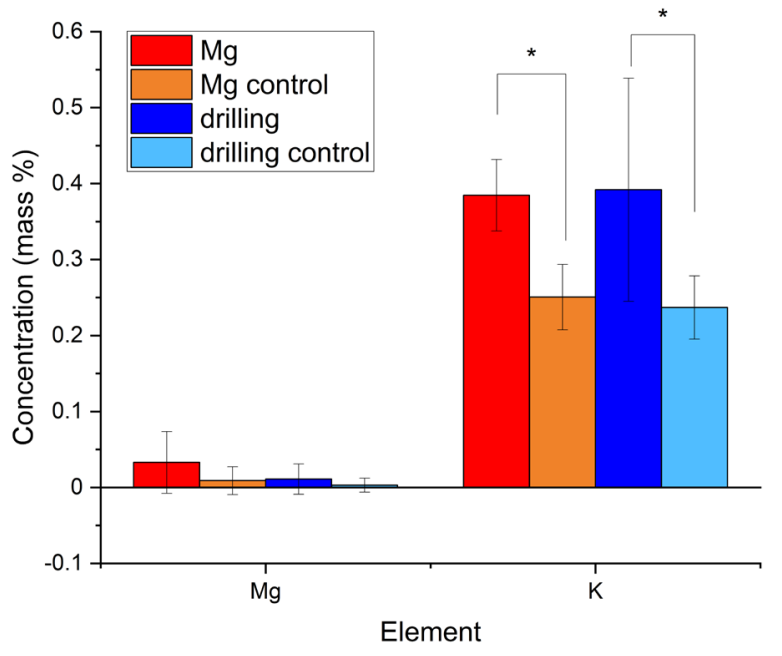

Fig. 13. Determination of $\mathrm{Mg}$ and $\mathrm{K}$ at the implant, obtained by imaging of bone-implant samples. Calculated mass \% represents the mean element intensities in the implant area. Mean \pm standard deviation; $n=10, * p<0.05$ Mann-Whitney. 
the tibia might be due to different bone structures. The SBP in the femur is thinner (Bhatla et al., 2018) and the amount of bone marrow in the area larger, which might have led to a better reactivity of the bone to the implanted $\mathrm{Mg}$, especially in the first time of degradation. However, in the light of bone quality, not only BV but BD is a crucial factor. As described earlier, often a sclerotic bone that is developed as a reaction to OA-induced stress (Burr, 2004), is less mineralised and of lower stability. In the present study, differences between both groups regarding BD were not significant. Although the BD in the drilling group 8 weeks after surgery exceeded that of the $\mathrm{Mg}$ group by $60 \%$, both values were in the range of normal $\mathrm{BD}$ and appeared to increase only slightly (1.5\% and $2.4 \%$, respectively). The higher reactivity of the femur regarding $\mathrm{BV}$ and tr.th. could also be found for the BD. The slight decrease in density was more in both groups from scan 1 to scan 2, while the increase between scan 2 and scan 3 was nearly the same as in the femur, summing up to a minimally decreased $\mathrm{BD}$ of the proximal tibia over the time. Considering the massive new bone formation especially in the $\mathrm{Mg}$ group, the quality of the new built bone could be judged positively.

An increased Ca content of the mineralised structure could not be observed utilising elemental imaging. However, differences in the $\mathrm{K}$ level comparing operated and non-operated control legs were shown. The observed increase in $\mathrm{K}$ in the animal groups subjected to mechanical treatment (drills) was comparable with and without $\mathrm{Mg}$ application, This increase could be insofar attributed to the procedure and probably related repair mechanisms.

The hypothesis that small, implanted $\mathrm{Mg}$ cylinders increased the mineralisation of the SBP and underlying trabeculae could be proven to be correct. However, the second part of the hypothesis that this enhancement of bone quality also influenced positively the cartilage could not yet be proven.

The histological assessment of cartilage could not reveal differences between samples of animals with implanted $\mathrm{Mg}$ and those of the drilling group. In comparison to the bone where the effect of $\mathrm{Mg}$ is well known and shown also in the present study, the cartilage was not as reactive. Once destroyed, the cartilage is very difficult to heal (Makris et al., 2015). Without external factors, the aim of cartilage regeneration has not yet been reached. Recently, defects have been treated by the transplantation of chondroblast/autologous, in-vitro-bred cartilage samples. While clinical outcomes in the long-term are very promising, there are still several drawbacks to these techniques. Recovery times are longer compared to microfracture (Kon et al., 2011) and load has to be applied carefully due to the vulnerability of the neotissue after surgery (Makris et al., 2015). The integration of the transplanted tissue into the existing cartilage depends on multiple factors including adequate blood supply to provide sufficient cell precursors and endogenous growth factors as well as mechanical stimuli to which chondrocytes react with proliferation and production of extracellular matrix (Appelman et al., 2011). Therefore, the chosen follow-up might have been too short to show effects on the cartilage itself. However, the detected bone changes were expected to influence the overlying cartilage. For a reliable assessment of this effect, a longer time point after implantation of the cylinders should have been examined as well as a larger number of animals. The most interesting result of the histological assessment was that the present pilot study could reveal a significant difference between the left, non-operated control samples. Animals of the $\mathrm{Mg}$ group showed a significantly better state of cartilage than animals of the drilling group. Altered loading of the contralateral limb is described in the literature (Metcalfe et al., 2013) and cartilage lesions in OA patients in the contralateral joint are very likely to develop (Metcalfe et al., 2013), with a gravity corresponding to the stage of the disease. The main reason is an increased load bearing of the contralateral limb to avoid pain, inducing load of the OA joint. Generally, experience of pain during OA is differently reported and its degree is not necessarily consistent with the degree of pathological changes (Lakkireddy et al., 2015). However, pain is one of the fundamental patient stress symptoms; therefore, its reduction is an important goal of OA treatment. The found increased cartilage lesions of the contralateral knee in the drilling group could be a sign for a higher pain sensitivity of the operated knee. However, no difference in either active or passive pain score could be found between $\mathrm{Mg}$ and drilling group despite the present structural changes shown by $\mu \mathrm{CT}$ and histology. A more sensitive diagnostic tool especially to assess active pain could clarify the relation between both parameters.

There could be a direct effect of $\mathrm{Mg}$ on the cartilage. Yue et al. (2019) showed that high concentrations of $\mathrm{Mg}$ inhibit the calcification of extracellular matrix in an ADCT5 cell line. Additionally, they found that high concentrations of $\mathrm{Mg}^{2+}$ ions have a protective effect in vivo on the cartilage of mice that suffered from OA after destabilisation of the medial meniscus (Yue et al., 2019). Therefore, beside the impact on the bone structure, implantation of Mg implants might have a secondary effect by directly protecting the cartilage itself due to increased $\mathrm{Mg}^{2+}$ ion concentration in the synovial fluid. However, examination of this was not part of the present study but could be implemented in further ones.

\section{Summary and conclusion}

Implanted, small Mg-cylinders significantly increased $\mathrm{BV}$ in the femoral condyles in comparison to solely drilling holes, as observed in an 8-week study. Simultaneously, BD value was nearly constant over time. Thus, $\mathrm{Mg}$ cylinders could improve the quality of the SBP, which possibly could prevent further OA 
progression. The fact that the cartilage of the nonoperated contralateral knee showed significantly more pathological changes in the drilling group indicated that the implanted $\mathrm{Mg}$ cylinders might have had a positive influence on the perception of pain, which resulted in a more even distribution of load bearing between both limbs. Further studies should examine the impact of implanted $\mathrm{Mg}$ cylinders in $\mathrm{OA}$ on pain using relevant biomarkers (e.g. nerve growth factor) and more sensitive methods for gait analysis (e.g. pressure measurement using a sensor mat as well as longer follow-up periods to focus on the progression of cartilage pathology). Furthermore, different alloying elements or the number of implanted cylinders would most likely have a different impact on bone and cartilage and should be considered.

\section{References}

Acar N, Balkarli H, Soyuncu Y, Ozbey O, Celik-Ozenci C, Korkusuz P, Ustunel I (2016) The determination of apoptosis rates on articular cartilages of ovariectomized rats with and without alendronate treatment. Histol Histopathol 31: 635645 .

Ahmad Agha N, Willumeit-Romer R, Laipple D, Luthringer B, Feyerabend F (2016) The degradation interface of magnesium based alloys in direct contact with human primary osteoblast cells. PLoS One 11: e0157874. DOI: 10.1371/journal.pone.0157874.

Alaneme KK, Okotete EA (2017) Enhancing plastic deformability of $\mathrm{Mg}$ and its alloys - A review of traditional and nascent developments. J magnesium alloys 5: 460-475.

Appelman TP, Mizrahi J, Elisseeff JH, Seliktar D (2011) The influence of biological motifs and dynamic mechanical stimulation in hydrogel scaffold systems on the phenotype of chondrocytes. Biomaterials 32: 1508-1516.

Arden N, Nevitt MC (2006) Osteoarthritis: epidemiology. Best Pract Res Clin Rheumatol 20: 3-25.

Bellido M, Lugo L, Roman-Blas JA, Castaneda S, Calvo E, Largo R, Herrero-Beaumont G (2011) Improving subchondral bone integrity reduces progression of cartilage damage in experimental osteoarthritis preceded by osteoporosis. Osteoarthritis Cartilage 19: 1228-1236.

Bobinac D, Marinovic M,Bazdulj E, Cvijanovic O, Celic T, Maric I, Spanjol J, Cicvaric T (2013) Microstructural alterations of femoral head articular cartilage and subchondral bone in osteoarthritis and osteoporosis. Osteoarthritis Cartilage 21: 1724-30.

Bhatla JL, Kroker A, Manske SL, Emery CA, Boyd SK (2018) Differences in subchondral bone plate and cartilage thickness between women with anterior cruciate ligament reconstructions and uninjured controls. Osteoarthritis Cartilage 26: 929-939.

Burr DB (2004) Anatomy and physiology of the mineralized tissues: role in the pathogenesis of osteoarthrosis. Osteoarthritis Cartilage 12 Suppl A: S20-30.

Carreau A, El Hafny-Rahbi B, Matejuk A, Grillon C, Kieda C (2011) Why is the partial oxygen pressure of human tissues a crucial parameter? Small molecules and hypoxia. J Cell Mol Med 15: 1239-1253.

Coyle CH, Izzo NJ, Chu CR (2009) Sustained hypoxia enhances chondrocyte matrix synthesis. J Orthop Res 27: 793-799.

Dobroň P, Chmelík F, Yi S, Parfenenko K, Letzig D, Bohlen J (2011) Grain size effects on deformation twinning in an extruded magnesium alloy tested in compression. Scripta Mater 65: 424-427.

Grünewald TA, Rennhofer H, Hesse B, Burghammer M, Stanzl-Tschegg SE, Cotte M, Löffler JF, Weinberg AM, Lichtenegger HC (2016) Magnesium from bioresorbable implants: distribution and impact on the nano- and mineral structure of bone. Biomaterials 76: 250-260.

Grynpas MD, Alpert B, Katz I, Lieberman I, Pritzker KP (1991) Subchondral bone in osteoarthritis. Calcif Tissue Int 1: 20-26.

Helmholz H, Luthringer-Feyerabend BJC, Willumeit-Römer R (2019) Elemental mapping of biodegradable magnesium-based implants in bone and soft tissue by means of $\mu \mathrm{X}$-ray fluorescence analysis. J Anal Atom Spectrom 34: 356-365.

Jahn K, Saito H, Taipaleenmaki H, Gasser A, Hort N, Feyerabend F, Schlüter H, Rueger JM, Lehmann W, Willumeit-Römer R, Hesse E (2016) Intramedullary Mg2Ag nails augment callus formation during fracture healing in mice. Acta Biomater 36: 350-360.

Janning C, Willbold E, Vogt C, Nellesen J, MeyerLindenberg A, Windhagen $\mathrm{H}$, Thorey F, Witte $\mathrm{F}$ (2010) Magnesium hydroxide temporarily enhancing osteoblast activity and decreasing the osteoclast number in peri-implant bone remodelling. Acta Biomater 6: 1861-1868.

Kon E, Filardo G, Berruto M, Benazzo F, Zanon G, Della Villa S, Marcacci M (2011) Articular cartilage treatment in high-level male soccer players: a prospective comparative study of arthroscopic secondgeneration autologous chondrocyte implantation versus microfracture. Am J Sports Med 39: 2549-2557.

Kraus T, Fischerauer SF, Hanzi AC, Uggowitzer PJ, Loffler JF, Weinberg AM (2012) Magnesium alloys for temporary implants in osteosynthesis: in vivo studies of their degradation and interaction with bone. Acta Biomater 8: 1230-1238.

Kree, V, Bohlen, J, Letzig, D, Kainer, KU (2004) The metallographical examination of magnesium alloys. Practical Metallography 41: 233-246.

Lakkireddy M, Bedarakota D, Vidyasagar J, Rapur S, Karra M (2015) Correlation among radiographic, arthroscopic and pain criteria for the diagnosis of knee osteoarthritis. J Clin Diagn Res 9: RC04-7.

Laverty S, Girard CA, Williams JM, Hunziker EB, Pritzker KP (2010) The OARSI histopathology initiative-recommendations for histological assessments of osteoarthritis in the rabbit. Osteoarthritis Cartilage 18 Suppl 3: S53-65. 
Makris EA, Gomoll AH, Malizos KN, Hu JC, Athanasiou KA (2015) Repair and tissue engineering techniques for articular cartilage. Nat Rev Rheumatol 11: 21-34.

Martinez Sanchez AH, Luthringer BJ, Feyerabend F, Willumeit R (2015) Mg and Mg alloys: how comparable are in vitro and in vivo corrosion rates? A review. Acta Biomater 13: 16-31.

Metcalfe AJ, Stewart C, Postans N, Dodds AL, Holt CA, Roberts AP (2013) The effect of osteoarthritis of the knee on the biomechanics of other joints in the lower limbs. Bone Joint J 95: 348-353.

Nidadavolu EPS, Feyerabend F, Ebel T, WillumeitRomer R, Dahms M (2016) On the determination of magnesium degradation rates under physiological conditions. Materials (Basel) 9: 627. DOI: 10.3390/ ma9080627.

Nygren H, Bigdeli N, Ilver L, Malmberg P (2017) Mg-corrosion, hydroxyapatite, and bone healing. Biointerphases 12: 02C407. DOI: 10.1116/1.4982601.

Oliver-Welsh L, Griffin JW, Meyer MA, Gitelis ME, Cole BJ (2016) Deciding how best to treat cartilage defects. Orthopedics 39: 343-350.

Pauly HM, Larson BE, Coatney GA, Button KD, DeCamp CE, Fajardo RS, Haut RC, Haut Donahue TL (2015) Assessment of cortical and trabecular bone changes in two models of post-traumatic osteoarthritis. J Orthop Res 33: 1835-1845.

Shen Y, Liu W, Wen C, Pan H, Wang T, Darvell BW, Lu WW, Huang W (2012) Bone regeneration: importance of local $\mathrm{pH}$-strontium-doped borosilicate scaffold. J Mater Chem 22: 8662-8670.

van Zuijlen MA, Vrolijk PWF, van der Heyden, MAG (2010) Bilateral successive cranial cruciate ligament rupture treated by extracapsular stabilization surgery in a pet rabbit (oryctolagus cuniculus). J Exot Pet Med 19: 245-248.

Willbold E, Weizbauer A, Loos A, Seitz JM, Angrisani N, Windhagen H, Reifenrath J (2017) Magnesium alloys: a stony pathway from intensive research to clinical reality. Different test methods and approval-related considerations. J Biomed Mater Res A 105: 329-347.

Witte F, Kaese V, Haferkamp H, Switzer E, MeyerLindenberg A, Wirth CJ, Windhagen H (2005) In vivo corrosion of four magnesium alloys and the associated bone response. Biomaterials 26: 3557-3563.

Yu W, Li R, Long J, Chen P, Hou A, Li L, Sun X, Zheng G, Meng H, Wang Y, Wang A, Sui X, Guo Q, Tao S, Peng J, Qin L, Lu S, Lai Y (2019) Use of a three-dimensional printed polylactidecoglycolide/tricalcium phosphate composite scaffold incorporating magnesium powder to enhance bone defect repair in rabbits. J Orthop Translat 16: 62-70.

Yue J, Jin S, Gu S, Sun R, Liang Q (2019) High concentration magnesium inhibits extracellular matrix calcification and protects articular cartilage via Erk/autophagy pathway. J Cell Physiol 234: 2319023201.

Zeller-Plumhoff B, Malich C, Kruger D, Campbell G, Wiese B, Galli S, Wennerberg A, Willumeit-
Römer R, Wieland F (2020) Analysis of the bone ultrastructure around biodegradable $\mathrm{Mg}-\mathrm{xGd}$ implants using small angle $x$-ray scattering and $x$-ray diffraction. Acta Biomater 101: 637-645.

\section{Web Reference}

1. https:/www.who.int/news-room/fact-sheets/ detail/obesity-and-overweight [23.08.2021]

\section{Discussion with Reviewer}

Reviewer: Are you expecting similar results by using an $\mathrm{Mg}$ alloy? Which elements do you expect to have higher influence on the implant performance?

Authors: Changing the implanted components, as suggested, by using e.g. a different $\mathrm{Mg}$ alloy would also change the results. Alloying elements influence different characteristics of the implants such as degradation rate, biocompatibility or mechanical properties (Angrisani et al., 2012; Hühnerschulte et al., 2012; Kraus et al., 2012; Wolters et al., 2013, additional references). While mechanical properties are of less interest for the application examined in the present study, biocompatibility and degradation rate are important. Higher degradation rates lead to more gas generation, which in turn could have a negative impact on the surrounding bone or even the formation of bony lacunae (Wolters et al., 2013, additional reference). Furthermore, the density of the adjacent bone tissue could be affected (Angrisani et al., 2012, additional reference). Also, biocompatibility and degree of osteoconductivity are different depending on the alloying elements which was shown e.g. by Hühnerschulte et al. (2012, additional reference) and also very impressively by Kraus et al. (2012). In addition, unwanted impurities could lead to an accelerated degradation and therewith higher impact. Here, especially Fe is noteworthy since its effect is relatively strong.

Reviewer: Why did you select the bead shape?

Authors: The aim was to distribute the Mg-based implants evenly within the subchondral bone. The size of the single implants should be small enough so that the defects were comparable to the solely drilled holes to allow for an exact analysis between the two groups. Regarding the shape, for example, tantalum beads are used for the diagnosis of implant loosening after hip arthroplasty. Such beads served as a model for the current study. However, during final decision on implant design, geometry of real beads was changed to small cylinders, since production was more standardisable and different sizes were easier to scale and produce. If we would like to increase the implanted amount of $\mathrm{Mg}$, the cylindrical implants could be very easily made longer, instead of having bigger beads or implanting more beads. Furthermore, 
implantation into the bone could be easily realised as described in the present study. Therefore, small-sized cylinders represented the most promising approach.

Reviewer: What do you expect to be the effect of implant's shape and size on subchondral bone quality?

Authors: Both shape and size of the implant influence its degradation. Surface characteristics (morphology, area/ratio) could alter the degradation rate. The size of the implants determines the amount of components released. A faster degradation would lead to more gas, which in turn could induce bone resorption. Smaller implants would dissolve faster and therewith have a shortened time of impact, whereas bigger implants would have a prolonged time of impact. The most favoured implant would degrade homogeneously, with a moderate degradation rate and over a sufficient time. However, the optimal time until total degradation could not be determined. Although the impact on the subchondral bone seemed to be good, no effect was shown on the cartilage itself. Furthermore, it should be examined how the subchondral bone further remodels after total degradation of the implants.

\section{Additional References}

Angrisani N, JM Seitz JM, Meyey-Lindenberg A, Reifenrath J (2012) Rare earth metals as alloying components in magnesium implants for orthopaedic applications. Intech Open. DOI: 10.5772/48335.

Hühnerschulte T, Reifenrath J, von Rechenberg B,Dziuba D, Seitz, J-M, Bormann D, Windhagen H, Meyer-Lindenberg (2012) A In vivo assessment of the host reactions to the biodegradation of the two novel magnesium alloys ZEK100 and AX30 in an animal model. Biomedical Engineering Online. DOI: 10.1186/1475-925X-11-14.

Wolters L, Angrisani N, Seitz J, Helmecke P, Weizbauer A, Reifenrath J (2013) Applicability of degradable magnesium lae442 alloy plate-screwsystems in a rabbit model. Biomed Tech (Berl) 58 Suppl 1. DOI: 10.1515/BMT-2013-4059.

Editor's note: The Scientific Editor responsible for this paper was Mauro Alini. 